Research Article

\title{
Asymptomatic Malaria and Helminths Coinfection and Its Association with Anemia among Primary School Children in Gedeo Zone, Southern Ethiopia: A Cross-Sectional Study
}

\author{
Feven Wudneh $\mathbb{D}^{1},{ }^{1}$ Yabibal Gebeyehu, ${ }^{2}$ and Sara Anberbir ${ }^{1}$ \\ ${ }^{1}$ Department of Medical Laboratory, College of Medicine and Health Sciences, Dilla University, Dilla, Ethiopia \\ ${ }^{2}$ School of Medicine, College of Medicine and Health Sciences, Dilla University, Dilla, Ethiopia \\ Correspondence should be addressed to Feven Wudneh; fevenwudneh@gmail.com
}

Received 8 May 2021; Accepted 7 August 2021; Published 13 September 2021

Academic Editor: Ran Wang

Copyright $\odot 2021$ Feven Wudneh et al. This is an open access article distributed under the Creative Commons Attribution License, which permits unrestricted use, distribution, and reproduction in any medium, provided the original work is properly cited.

\begin{abstract}
Background. Asymptomatic malaria and helminths coinfection occurs mainly in the tropics and subtropics where poverty and sanitary practice favor its high prevalence. In the tropics, where malaria is endemic, helminths also thrive resulting in coinfection. This study aimed to access the prevalence of asymptomatic malaria and helminths coinfection and its contribution for anemia in primary school children of Gedeo Zone, Southern Ethiopia. Methodology. This was a cross-sectional study conducted among 413 primary school children from February to April 2020. Finger-prick blood samples were used to determine asymptomatic malaria and hemoglobin concentrations. Stool samples were collected and processed through formalin-ether concentration techniques to detect the presence of intestinal helminths. Data were double entered into Epi Data version 3.1 software and exported to SPSS version 20 for analysis. Pearson's chi-square and correlation analysis were performed as part of the statistical analyses. Result. A total of 413 primary school children aged 6 to 16 years (mean age \pm SD: $10.7 \pm 2.64$ years) were enrolled in the study. 159 (38.5\%) of school children were infected with at least one of the parasitic diseases. The overall prevalence of asymptomatic malaria and intestinal helminths was $46(11.1 \%)$ and 113 (27.3\%) respectively. Asymptomatic malaria and helminths coinfection was 29 (7\%). Total of $39.1 \%$ of asymptomatic malaria-infected school children were anemic, which is statistically significant $(P<0.05) .15 .9 \%$ of helminths-infected school children were anemic, not statistically significant $(P>0.05)$. The prevalence of anemia was $12(41.3 \%)$ among coinfected students, which is statistically significant $(P<0.005)$. Conclusion. Asymptomatic malaria and helminths coinfection affects the health status of considerable number of primary school children in the study area. Therefore, simultaneous combat against the two parasitic infections is crucial to improve health of the school children.
\end{abstract}

\section{Introduction}

Asymptomatic malaria and helminths infection are among the most prevalent diseases in sub-Saharan Africa. It is estimated that over one-third of the world's population, mainly those individuals living in the tropics and subtropics, are infected by intestinal helminths or one or more of Plasmodium species [1]. Both intestinal helminthic and malarial infection cause high rates of morbidity. Climatic conditions, poverty, and sanitary practices favor their high rate of prevalence in developing countries [2].

Although there is no standard definition for "asymptomatic" malaria infections, it is generally accepted to be malarial parasitemia of any density, in the absence of fever or other acute symptoms in individuals who have not received recent antimalarial treatment [3]. There are about 300-500 million incidences of malaria causing 2-3 million deaths each year in the tropical and subtropical regions of the world [4]. About $90 \%$ of these deaths occur in sub-Saharan Africa [4]. In Ethiopia, the burden of malaria continues to cause a substantial number of morbidity and mortality which accounts for most outpatient visits. Malaria has been one of the main causes of hospitalization and deaths in the country. About $60 \%$ of the population are living in the malaria-risky areas, mainly areas that lie below $2000 \mathrm{~m}$ above sea level. However, several pockets with microepidemiological 
conditions supporting malaria transmission occur in areas above this altitude. Plasmodium falciparum and $P$. vivax are the dominant parasites responsible for the majority of malaria cases in Ethiopia [5-7], and malaria is ranked as the leading communicable disease accounting for about $30 \%$ of the overall disability adjusted life years lost [8].

Intestinal helminths are also the cause of malnutrition, or aggravate existing malnutrition; through damage to the gastrointestinal mucosal epithelium and inflammation, reduced food intake, malabsorption, and increased nitrogen loss in faeces and diarrhea, malaria-helmiths coinfections further compromise the health condition of the patient [9-12].

In Ethiopia, like other developing countries, infections with the major soil-transmitted helminths, including Ascaris lumbricoides, Trichuris trichiura, Hook worm, and Hymenolophis nana, are widely spread with variable prevalence [13-15].

Asymptomatic malaria and helminths coinfection overlapping distribution contributes substantially to anemia [16]. Malaria in endemic regions causes anemia through mechanisms such as haemolysis, increased spleen clearance of infected and uninfected red blood cells and cytokineinduced dyserythropoesis, destruction of parasitized red blood cells, shortening of the life span of nonparasitized red blood cells, and decreased production of red blood cells in the bone marrow [2]. Mechanisms of anemia include lysis and phagocytosis of infected red blood cells while depending on the intensity of the malaria infection [17]. An asymptomatic malaria host serves as a reservoir for the malaria parasite, and asymptomatic malaria is now recognized as an important obstacle to malaria elimination [18]. Asymptomatic malaria and helminths coinfection greatly affects the socioeconomic development of communities in multiple ways. From health perspective, it affects the physical and mental wellbeing of school children, thereby leading to increased absenteeism and retarded cognitive development, and thus, learning disabilities although the literature is inconclusive [10,19]. Most importantly, helminths complicate the clinical picture of more serious diseases such as malaria and cause anemia as a result of direct blood loss, nutritional theft, and impairment of appetite due to immunological factors.

There is evidence that Ascaris lumbricoides-associated vitamin A deficiency may further increase the risk of anemia in those coinfected with malaria [18]. Hookworms are the most pathogenic because of their propensity to feed on blood [20].

This parasitic disease affects the health status of the community in sub-Saharan Africa. Also, studies indicated that individuals coinfected with more than one parasite species are at risk of increased morbidity, as well as at a risk of developing frequent and more severe disease due to interactions among the infecting parasite species [21, 22]. However, how concurrent infections affect the epidemiology and/or the pathogenesis of each other remains controversial ranging from increased severity of malaria to reduced severity and incidence of malaria during helminths coinfection [23-25].
Therefore, this study aimed to assess the prevalence of intestinal helminths and asymptomatic malaria and its effects on anemia among primary school children. The results of this study could help concerned stakeholders to take action on the prevention of intestinal helminths and asymptomatic malaria.

\section{Materials and Methods}

2.1. Study Design and Period. A cross-sectional study was conducted from February to April 2020 in selected Dilla town and Dilla Zuria woreda primary schools.

2.2. Study Area. Dilla is located $360 \mathrm{~km}$ to the south of Addis Ababa, the capital of Ethiopia, with an area of $1123.47 \mathrm{sq} . \mathrm{kms}$. It is located $5^{\circ} 53^{\prime} \mathrm{N}$ to $6^{\circ} 27^{\prime} \mathrm{N}$ latitude and $38^{\circ} 8^{\prime}$ to $38^{\circ} 30^{\prime}$ east longitude. Dilla town and Dilla Zuria woreda are located in Gedeo zone (Figure 1). The altitude of the zone ranges from 1268 to $2993 \mathrm{~m}$ above sea level (masl). The mean annual temperature is between $12.6^{\circ} \mathrm{C}$ and $30^{\circ} \mathrm{C}$. The mean annual rainfall ranges from 1001 to $1800 \mathrm{~mm}$, and the pattern is bimodal, with a short rain season between March and May accounting for 30\% of total rain fall. The long rain season is between July and October accounting for more than $60 \%$ of the total rainfall.

2.2.1. Study Population. All primary school children aged 5 to 16 years attending class in Dilla town and Dilla Zuria woreda and who did not take antimalarial and antihelminths drug two weeks prior to the study commencing were considered as the study population.

2.2.2. Sampling Techniques. There are 31 primary schools in Dilla and Dilla Zuria woreda. Six of them were selected by simple random sampling and by considering proportional allocation: three primary schools (Kirinchaf, Dawit, and Kofe) and another three primary schools (Sisota, Aroresa, and Chichu) were selected from Dilla and Dilla Zuria woreda, respectively. Finally, representative primary school children were selected by systematic random sampling techniques from the registration list of selected schools.

2.2.3. Sample Size Determination. Sample size was calculated using a single population proportion formula by taking a previous similar study conducted in Alaba kulito [12], 95\% confidence interval, 5\% margin of error, and 10\% nonresponse rate ( 38 students), and a total of 417 participants were enrolled in the study.

2.2.4. Data Collection and Study Sample. Sociodemographic data were collected using pretested structured questionnaires prepared in local languages (Gede'offa and Amharic). The study participants were also interviewed to obtain sociodemographic characteristics and other information (age, sex, hand wash practice after toilet use, shoe wearing habit, source of drinking water, and residence). A total of 417 primary school children were enrolled in the study, and 


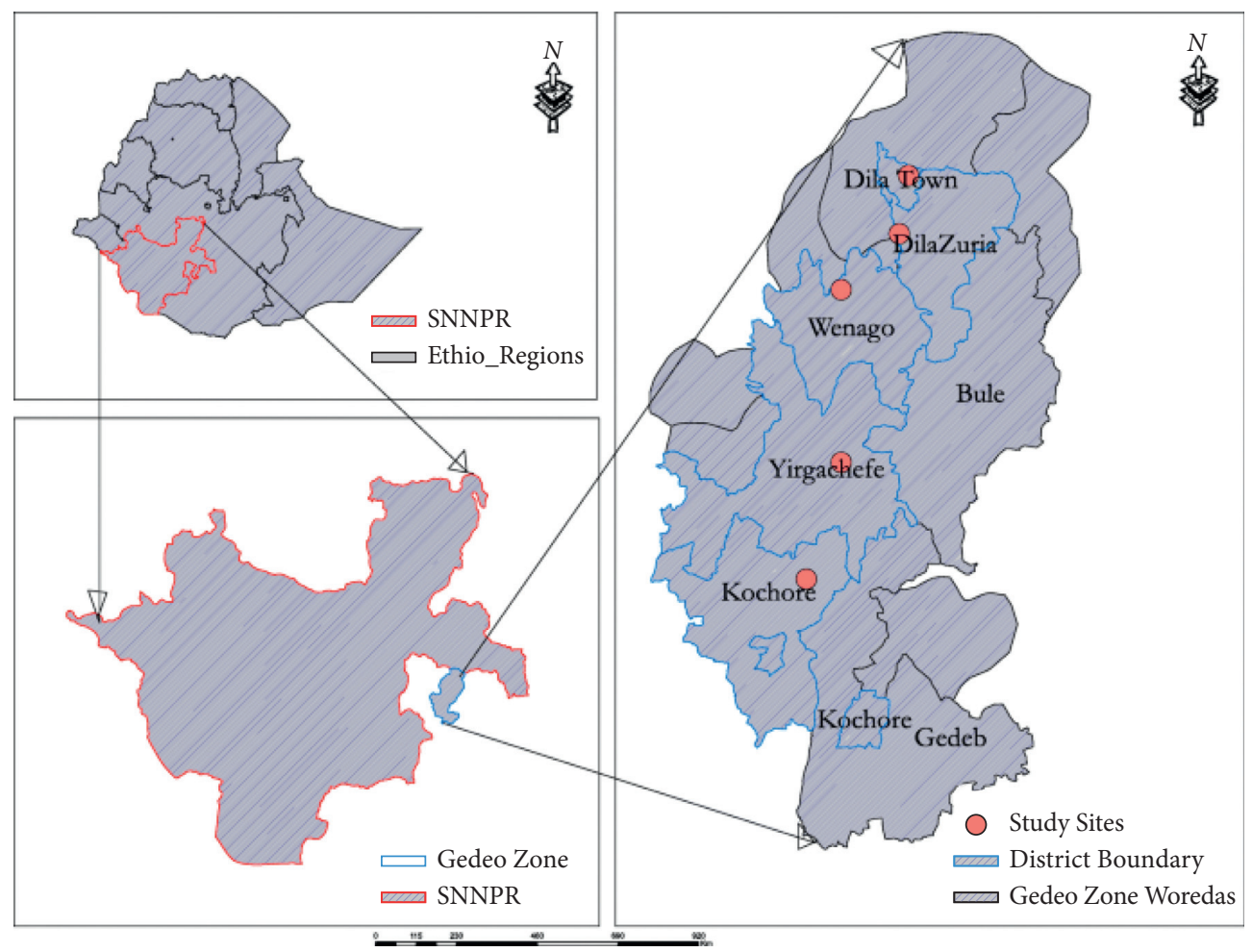

Figure 1: Map of Gedeo zone.

three of them were excluded from the study since they did not fulfill the inclusion criteria. Therefore, a total of 413 primary school children participated in the study.

\subsubsection{Blood Film Preparation and Examination of Malaria} Parasites. The clean slides were labeled with patient identification number, date, and time of collection at the frosted end. The blood sample was collected from the middle or ring finger after cleaning with a 70\% alcohol-moistened swab and dried with a piece of dry cotton after pricked using a disposable blood lancet. Through wiping off the first drop of blood, $6 \mu \mathrm{l}$ of blood for thick and $2 \mu \mathrm{l}$ of blood for thin blood films were collected $[22,26]$; then; slides were air-dried and fixed by using absolute methanol for 30 seconds before staining. Staining was carried out using $10 \%$ Giemsa solution for 10 minutes. The stained slides were rinsed with water, air-dried, and finally, examined using a microscope. To avoid false positive and negative result, microscopic examination was performed by two experts while discrepancies were resolved by a third opinion $[27,28]$. Smears were reported as negative after observing at least 100 fields using high-power fields (oil immersion).

2.2.6. Formalin-Ether Concentration Method. A stool sample was collected from each participant using a clean, leakproof, and sterile stool cup and preserved using $10 \%$ formalin and then processed by the formalin-ether concentration procedure; $2 \mathrm{ml}$ of preserved stool was added in a clean conical centrifuge tube containing $7 \mathrm{ml}$ of $10 \%$ formol water. The suspension was filtered through a sieve into a
$15 \mathrm{ml}$ conical centrifuge tube. Then, $4 \mathrm{ml}$ of diethyl ether was added to the formalin solution and the content was centrifuged at $300 \mathrm{rpm}$ for 1 minute. The supernatant was discarded, and stool smears were prepared on sterile slides from the sediment and then three slides were prepared for each participant $[22,29]$. Finally, the slides were microscopically examined by two experts while discrepancies were resolved by a third opinion under a magnification power of 10x and 40x objective to identify intestinal helminths.

2.2.7. Hemoglobin Concentration. Hemoglobin concentration was determined using a Hemocue Hb 201 analyser $[30,31]$. Anemia classification was interpreted by the WHO anemia classification. According to the WHO guidelines, for children 5-11 years of age, hemoglobin levels of $11-11.4 \mathrm{~g} / \mathrm{dl}$ were considered as mild, $7-10.9 \mathrm{~g} / \mathrm{dl}$ moderate, and $<7 \mathrm{~g} / \mathrm{dl}$ severe anemia and for children 12-16 years of age, the hemoglobin level $11-11.9 \mathrm{~g} / \mathrm{dl}$ was considered as mild, $7-10.9 \mathrm{~g} / \mathrm{dl}$ moderate, and $<7 \mathrm{~g} / \mathrm{dl}$ severe anemia [32].

2.2.8. Data Quality Control. To keep the quality of data, onsite training was given for data collectors on how to collect sociodemographic data and other associated factors. Data were checked for completeness before and after entry. Reagent and instrument quality was also checked for expiry dates and any functional problems.

The slides were microscopically examined by two experts, while discrepancies were resolved by a third opinion for quality control. Similarly, quality control was performed on hemoglobin measures, Hemocue Hb $201+$ analyser and 
Mission ${ }^{\circledR}$ Hemoglobin Plus Hb (an automated haematology analyser).

2.3. Statistical Analysis. The data were double entered to EpiData software and exported to SPSS version 20 software for analysis. Descriptive statistics was performed for sociodemographic characteristics. Pearson's chi-square and multivariate logistic regression association were also performed to assess the association between malaria/helminths coinfection and anemia. Statistical significance were considered if $P<0.05$.

2.4. Ethical Consideration. Ethical clearance was received from the research and ethical review committee of Dilla University. Before data collection, the objectives of the study were explained to the study participants, parents, and the school community. Written informed consent was obtained from partners or the legal guardians. Children infected with any of the parasites were referred to the nearby health institution for treatment. All information obtained from the study participants was coded to keep confidentiality.

\section{Results}

3.1. Sociodemographic Characteristics. A total number of 413 participants were enrolled in the study. About 322/413 (78\%) of the study participants were between the age range of 10-15 years. Two hundred and twenty-seven study participants (55\%) were males, 132 (32\%) used bed net, 362 $(87.5 \%)$ washed their hand before and after toilet use, and $305(73.8 \%)$ and 108 (26.2\%) of study participants wore shoe always and sometimes, respectively. About 374 (90.3\%) used toilet, and the rest 40 (9.7\%) used open space for defecation. The source of drinking water as pipe, well, and river was 322 (78\%), 84 (20.3\%), and 6 (1.5\%), respectively. Two hundred and seventeen $(51.8 \%)$ of them were living in urban areas (Table 1).

3.1.1. Prevalence of Asymptomatic Malaria. The overall prevalence of asymptomatic malaria was 46 (11.1\%), 21 (5.1\%) Plasmodium falciparum, 23 (5.6\%) Plasmodium vivax, and 2 $(0.4 \%)$ mixed infections. The majority, $29(7 \%)$ of malariainfected students were in the age group of 10-14 years. The prevalence of asymptomatic malaria among males and females was $21(5.1 \%)$ and 25 (6\%), respectively (Table 2), and higher prevalence was observed among rural students (Figure 2).

3.1.2. Prevalence of Intestinal Helminths Infection. Out of 413 children, $113(27.3 \%)$ were infected by at least one intestinal helminths. The prevalence of intestinal helminthic infection was higher, 85 (20.5\%), among the age group of 10-14 years. Ascaris lumbricoides was the most prevalent with 57 (13.8) (Table 3).

3.1.3. Asymptomatic Malaria and Helminths Coinfection. The prevalence of asymptomatic malaria and helminths coinfection was 29 (7\%). The magnitude of Ascaris
TABle 1: Sociodemographic characteristics of primary school children in Gedeo zone, Ethiopia.

\begin{tabular}{lccc}
\hline Variables & Category & $N$ & $\begin{array}{c}\text { Percent } \\
(\%)\end{array}$ \\
\hline \multirow{3}{*}{ Age } & $5-9$ & 61 & 14.8 \\
& $10-14$ & 322 & 78 \\
\multirow{2}{*}{ Sex } & $>15$ & 30 & 7.2 \\
\hline \multirow{2}{*}{ Residence } & $\mathrm{M}$ & 227 & 55 \\
& $\mathrm{~F}$ & 186 & 45 \\
\hline \multirow{2}{*}{ Religion } & Urban & 217 & 52.5 \\
& Rural & 196 & 47.5 \\
\hline \multirow{2}{*}{ Bed net use } & Orthodox & 98 & 23.6 \\
& Muslim & 33 & 8 \\
\multirow{2}{*}{ Source of drinking water } & Protestant & 282 & 68.4 \\
\hline Hand washing practice before & Yes & 132 & 32 \\
meal & No & 281 & 68 \\
\hline \multirow{2}{*}{ Habit of wearing shoe } & Pipe & 322 & 78 \\
& Well & 84 & 20.3 \\
Latrine use & Underground & 9 & 1.7 \\
\hline
\end{tabular}

lumbricoides, Hook worm, and Taenia species among malaria-infected students was 18 (4.3\%), 10 (2.5\%), and 1 $(0.2 \%)$, respectively (Table 4$)$.

Students of 10-14 years were the most affected group, 20 (69\%). Ascaris lumbricoides/plasmodium vivax and Ascaris lumbricoides/Plasmodium falciparum takes a major share. The prevalence of coinfection among males and females was $11(38 \%)$ and $18(62 \%)$, respectively (Table 5).

3.1.4. Haemoglobin Measurement. From 413 participants tested for anemia, $45(10.9 \%)$ were found to be anemic according to the WHO classification. The prevalence of anemia was significant among the age group of 5-9, $P$ value 0.004, and there was no significant association observed among sex groups, $P$ value 0.37 (Table 6).

Among 46 (11.1\%) asymptomatic malaria-infected students, $18(39.1 \%)$ were anemic, which is statistically significant $(P=0.037)$. However, among 113 (27.3\%) students infected with helminths, $18(15.9 \%)$ were anemic, not statistically significant $(P=0.58)$, and among 29 (7\%) malariahelminths coinfected students, $12(41.3 \%)$ were anemic, which is statistically significant $(P=0.003)$ (Table 7$)$.

\section{Discussion}

The overlapping distribution of asymptomatic malaria and intestinal helminths coinfection is the main cause of anemia. In this study, we have assessed the magnitude of asymptomatic malaria and helminths coinfection, as well as its effect on the hemoglobin level, among primary school children of Gedeo Zone, Southern Ethiopia. 
TABLE 2: The prevalence of asymptomatic malaria among age and sex groups of primary school children in Gedeo zone, Ethiopia.

\begin{tabular}{|c|c|c|c|c|}
\hline \multirow{2}{*}{ Variables } & \multirow[b]{2}{*}{ Plasmodium falciparum } & \multicolumn{3}{|c|}{ Asymptomatic malaria by microscopy $N(\%)$} \\
\hline & & Plasmodium vivax & Mixed infection & Total \\
\hline \multicolumn{5}{|l|}{ Age } \\
\hline $5-9$ & $6(1.5 \%)$ & $7(1.7)$ & $1(0.2)$ & $14(3.4)$ \\
\hline $10-14$ & $14(3.4)$ & $14(3.4)$ & $1(0.2)$ & $29(7)$ \\
\hline$>15$ & $1(0.2)$ & $2(0.5)$ & 0 & $3(0.7)$ \\
\hline \multicolumn{5}{|l|}{ Sex } \\
\hline M & $9(2.2)$ & $11(2.7)$ & $1(0.2)$ & $21(5.1)$ \\
\hline $\mathrm{F}$ & $12(2.9)$ & $12(2.9)$ & $1(0.2)$ & $25(6)$ \\
\hline Total & $21(5.1)$ & $23(5.6)$ & $2(0.4)$ & $46(11.1)$ \\
\hline
\end{tabular}

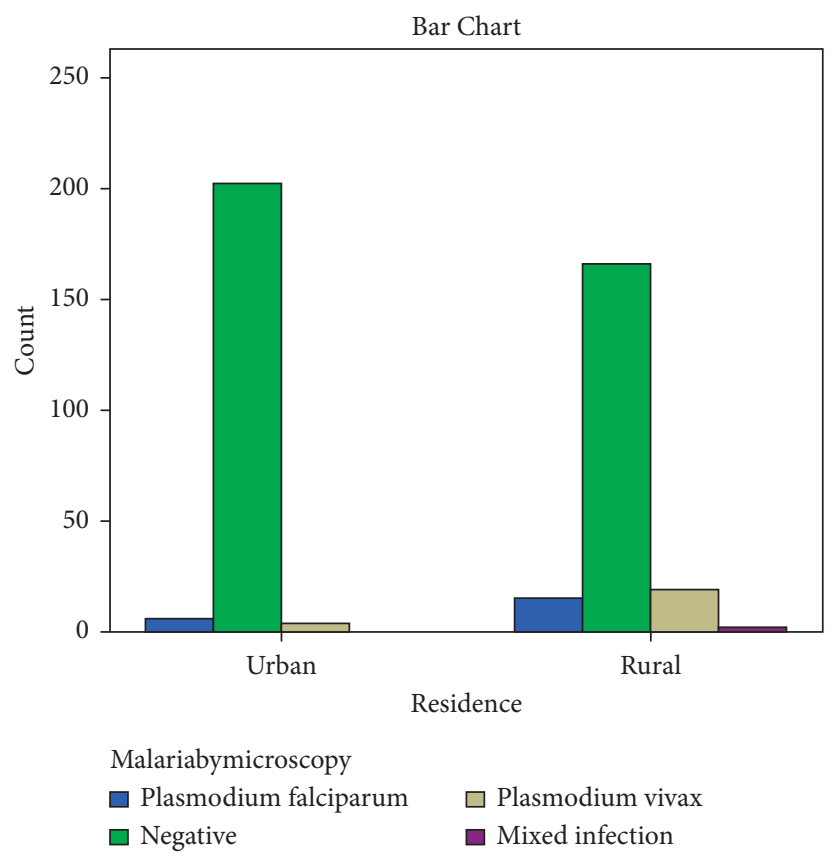

Figure 2: The prevalence of asymptomatic malaria among urban and rural residence of primary school children in Gedeo zone, Southern Ethiopia.

TABle 3: Prevalence of intestinal helminths among age and sex groups of primary school children in Gedeo zone, Ethiopia.

\begin{tabular}{|c|c|c|c|c|c|c|c|}
\hline & \multicolumn{5}{|c|}{ Prevalence of helminths $N(\%)$} & \multirow{2}{*}{ Total } & \multirow{2}{*}{$P$ value } \\
\hline & Ascaris lumbricoides & Tricuris tricura & Hook worm & H. nana & Taenia spp. & & \\
\hline \multicolumn{8}{|l|}{ Sex } \\
\hline $\mathrm{M}$ & $41(9.9)$ & $4(1)$ & $6(1.5)$ & $7(1.7)$ & $1(0.2)$ & $59(14.3)$ & \multirow{2}{*}{0.194} \\
\hline $\mathrm{F}$ & $36(8.7)$ & $4(1)$ & $9(2.1)$ & $5(1.2)$ & 0 & $54(13.1)$ & \\
\hline \multicolumn{8}{|l|}{ Age } \\
\hline $5-9$ & $14(3.4)$ & 0 & $5(1.2)$ & $1(0.2)$ & 0 & $20(4.8)$ & \\
\hline $10-14$ & $57(13.8)$ & $8(2)$ & $10(2.4)$ & $9(2.1)$ & $1(0.2)$ & $85(20.5)$ & 0.012 \\
\hline$>15$ & $6(1.4)$ & 0 & 0 & $2(0.5)$ & 0 & $8(1.93)$ & \\
\hline Total & $77(18.6)$ & $8(2)$ & $15(3.6)$ & $12(2.9)$ & $1(0.2)$ & $113(27.6)$ & \\
\hline
\end{tabular}

The prevalence of asymptomatic malaria among primary school children was 46 (11.1\%). The magnitude of Plasmodium vivax, Plasmodium falciparum, and mixed infection was $23(5.6 \%), 21(5.1 \%)$, and $2(0.5 \%)$, respectively. This finding is higher than that of the study conducted in Southern Ethiopia, 3.6\% [33], and
Democratic Republic, 6.3\% [17], and lower than that of the study reported from Ethiopia, 18.3\% [22], and Côte d' Ivoire, $50.3 \%$ [34]. The difference might be due to environmental variation, level of bed net distribution, seasonal variation, or the geographical difference of study participants. 
TABLE 4: Prevalence of asymptomatic malaria and helminths coinfection among primary school children in Gedeo zone, Southern Ethiopia.

\begin{tabular}{lcccc}
\hline \multirow{2}{*}{ Type of helminths } & \multicolumn{2}{c}{ Asymptomatic malaria infection, } & OR (95\% CI) \\
& Result & Positive & Negative & $P$ value \\
\hline \multirow{2}{*}{ A. lumbricoides } & Positive & $18(4.3)$ & $59(14.3)$ & $1.6(0.7-3.6)$ \\
\hline \multirow{2}{*}{ Hook worm } & Negative & $28(6.7)$ & $308(74.6)$ & 0.2 \\
\hline \multirow{2}{*}{ Taenia spp. } & Positive & $10(2.5)$ & $5(1.2)$ & $6(1.04-34.54)$ \\
\hline
\end{tabular}

TABLE 5: Prevalence of asymptomatic malaria and helminths coinfection among the age and sex group of primary school children in Gedeo zone, Southern Ethiopia.

\begin{tabular}{|c|c|c|c|c|c|c|}
\hline \multirow{2}{*}{ Variables } & \multicolumn{6}{|c|}{ Asymptomatic malaria and helminths coinfection by age and sex, $N(\%)$} \\
\hline & $\mathrm{AL}+\mathrm{PF}$ & $\mathrm{AL}+\mathrm{PV}$ & $\mathrm{HW}+\mathrm{PF}$ & $\mathrm{HW}+\mathrm{PV}$ & $\mathrm{PV}+$ Taenia & Total \\
\hline \multicolumn{7}{|l|}{ Age } \\
\hline $5-9$ & $1(3.45)$ & $3(10.3)$ & $1(3.45)$ & $1(3.54)$ & $1(3.45)$ & $7(24)$ \\
\hline $10-14$ & $6(20.6)$ & $6(20.6)$ & $3(10.3)$ & $5(17.2)$ & 0 & $20(69)$ \\
\hline$>15$ & $1(3.45)$ & $1(3.45)$ & 0 & 0 & 0 & $2(7)$ \\
\hline \multicolumn{7}{|l|}{ Sex } \\
\hline M & $2(6.9)$ & $4(13.8)$ & $1(3.45)$ & $3(10.34)$ & $1(3.45)$ & $11(38)$ \\
\hline $\mathrm{F}$ & $6(20.7)$ & $6(20.7)$ & $3(10.3)$ & $3(10.34)$ & & $18(62)$ \\
\hline Total & $8(27.5)$ & $10(34.5)$ & $4(13.8)$ & $6(20.7)$ & $1(3.45)$ & $29(100)$ \\
\hline
\end{tabular}

$\mathrm{PF}=$ P. falciparum $; \mathrm{PV}=P$. vivax $\mathrm{HW}=$ Hook worm; $\mathrm{AL}=$ Ascaris lumbricoides.

TAвLE 6: Anemia level among the age and sex group of primary school children in Gedeo zone, Southern Ethiopia.

\begin{tabular}{|c|c|c|c|c|c|}
\hline \multirow{2}{*}{ Variables } & \multirow{2}{*}{ Anemia, $N(\%)$} & \multirow{2}{*}{$P$ value } & \multirow{2}{*}{ AOR } & \multicolumn{2}{|c|}{ 95\% confidence interval } \\
\hline & & & & Lower bound & Upper bound \\
\hline \multicolumn{6}{|l|}{ Age } \\
\hline $5-9$ & $12(2.9)$ & \multirow{3}{*}{0.004} & \multirow{3}{*}{$\begin{array}{c}13.9 \\
2.3\end{array}$} & \multirow{3}{*}{$\begin{array}{l}2.4 \\
0.5\end{array}$} & \multirow{3}{*}{$\begin{array}{l}82.8 \\
10.9\end{array}$} \\
\hline $10-14$ & $31(7.5)$ & & & & \\
\hline$>15$ & $2(0.5)$ & & & & \\
\hline \multicolumn{5}{|l|}{ Sex } & \multirow{3}{*}{2.7} \\
\hline M & $24(5.8)$ & \multirow{2}{*}{0.14} & \multirow[t]{2}{*}{1.3} & \multirow[t]{2}{*}{0.6} & \\
\hline $\mathrm{F}$ & $21(5.1)$ & & & & \\
\hline Total & $45(10.9)$ & & & & \\
\hline
\end{tabular}

TABLE 7: Prevalence of anemia among asymptomatic malaria, helminths, and coinfected primary school children in Gedeo zone, Southern Ethiopia.

\begin{tabular}{lcccc}
\hline Type of infection & \multicolumn{2}{c}{ Anemia } & $P$ value & Total, $N(\%)$ \\
\hline Asymptomatic malaria & Absent (\%) & Present (\%) & $18(40)$ & 0.037 \\
Intestinal helminths & $27(60)$ & $18(15.9)$ & 0.58 & $113(27.4)$ \\
Coinfection & $95(84)$ & $12(41.3)$ & 0.003 & $29(7)$ \\
\hline
\end{tabular}

The prevalence of asymptomatic malaria decreased when the age of participants increased. The reason might be related with the development of protective immunity by repeated infections as well as acquisition of knowledge on malaria prevention and control mechanisms. A significant number of asymptomatic malaria cases were also observed in rural areas, which might be associated with suboptimal use of bed net. On the other hand, the prevalence of helminths was 113 $(27.6 \%)$ and it was lower than that of studies conducted in
Southern Ethiopia, 69\% [34], and Northwest Ethiopia, 49\% [35], and higher than that of the study conducted in North West Tigray, 12.7\% [36]. These might be related with schoolbased mass deworming campaigns conducted in the study area.

The prevalence of asymptomatic malaria and helminths coinfection was 29 (7\%). The prevalence of Ascaris lumbricoides coinfection with Plasmodium falciparum and Plasmodium vivax was 8 (1.9\%) and 10 (2.4\%), respectively. 
The prevalence Hookworm coinfection with Plasmodium falciparum and Plasmodium vivax was $4(1 \%)$ and $6(1.45 \%)$, respectively. This finding is lower than that of the study conducted in Southwest Nigeria [11], Côte d' Ivoire [34], and Ghana [16] and larger than that of the study in Nigeria [37]. This could be linked with environmental contamination, poor hand washing practice, insufficient latrine constriction, open air defecation, and small-scale distribution of bed net. A significant association of asymptomatic malaria and hook worm coinfection was observed. The coinfection had various underlying mechanisms including environmental, socioeconomic, and behavioural factors or due to involvement of immunological mechanisms which might lead to increased susceptibility of helminths-infected individuals for malaria infection.

The prevalence of anemia among asymptomatic malaria and helminths coinfected students was $41.3 \%$. This findings was higher than that of the study conducted in Northwest Ethiopia, 10.9\% [2], Southwest Nigeria, 34.4 [11], and Tanzania, 19.8 [29]. The result showed that the level of anemia among coinfected students was significantly higher. This might be associated with the blood loss mainly caused by the destruction of RBC and hook worm infection similar with the finding of the study conducted by Garcia LS [38].

\section{Conclusions}

Asymptomatic malaria and helminths coinfection was observed in the study area, and the prevalence of anemia was significantly associated with coinfection. Therefore, combined and integrated interventional measures should be taken including health education for school children about personal and environmental hygiene practices, use of bed net, encouraging footwear practice, and use of latrine.

\section{Abbreviations}

RBCs: Red blood cells

PF: $\quad$ Plasmodium falciparum

PV: $\quad$ Plasmodium vivax

STH: Soil-transmitted helminths

WHO: World Health Organization.

\section{Data Availability}

The data underlying this article are available in the article and in the supplementary materials.

\section{Additional Points}

In the currents study, we did not use advanced diagnostic techniques such as PCR and other advanced parasitological techniques. PCR is important to identify the gene which makes malaria asymptomatic and increase diagnostic accuracy and sensitivity. The other limitation of this study was we did not rule out other possible causes of anemia such as malnutrition.

\section{Ethical Approval}

Ethical clearance of the study was obtained from the institutional review board of Dilla University.

\section{Consent}

The objective of the study including the procedure followed, benefits, potential risks, and discomforts was explained to the participants. Informed written consent for children who participated in the study was taken from parents or legal guardians in both Amharic and Gede'offa language. Children infected with any of the parasitic infections were referred to the nearby health institution. Study identification number was used for identification, and information collected was kept confidential. Feedback for the study population in the form of dissemination workshops was conducted during the study. The children could withdraw at any time without penalty or loss of benefits. The proposal has been explained to the parents and children in the language they understand.

\section{Conflicts of Interest}

The authors declare no conflicts of interest.

\section{Authors' Contributions}

FW and YG conceived the study, participated in data collection and data analysis, and drafted and finalized the manuscript for publication. SA assisted in data collection and reviewed the initial and final draft of the manuscript. All authors read and approved the final manuscript.

\section{Acknowledgments}

The authors would like to acknowledge the Dilla University Research and Dissemination Office for funding and those primary school children participated in this study.

\section{Supplementary Materials}

The data of this article are given. (Supplementary Materials)

\section{References}

[1] N. Tuasha, E. Hailemeskel, B. Erko, and B. Petros, "Comorbidity of intestinal helminthiases among malaria outpatients of Wondo Genet health centers, southern Ethiopia: implications for integrated control," BMC Infectious Diseases, vol. 19, no. 1, p. 659, 2019.

[2] A. Alemu, Y. Shiferaw, A. Ambachew, and H. Hamid, "Malaria helminth co-infections and their contribution for aneamia in febrile patients attending Azzezo health center, Gondar, Northwest Ethiopia: a cross sectional study," Asian Pacific Journal of Tropical Medicine, vol. 5, no. 10, pp. 803809, 2012.

[3] I. Chen, S. E. Clarke, and R. Gosling, "Asymptomatic malaria: a chronic and debilitating infection that should be treated," PLoS Medicine, vol. 13, no. 1, Article ID e1001942, 2016.

[4] A. Simon-Oke, "Prevalence of malaria parasite among asymptomatic and symptomatic students of federal university 
of technology, Akure, Ondo State," British Journal of Research, vol. 4, p. 5, 2017.

[5] Federal Ministry of Health, Malaria Strategic Plan 2017-2020, Federal Ministry of Health, Addis Ababa, Ethiopia, 2017.

[6] S. Tesfaye, Y. Belyhun, T. Teklu, T. Mengesha, and B. Petros, "Malaria prevalence pattern observed in the highland fringe of Butajira, Southern Ethiopia: a longitudinal study from parasitological and entomological survey," Malaria Journal, vol. 10, no. 1, p. 153, 2011.

[7] Federal Ministry of Health, Malaria Elimination Road Map, Federal Ministry of Health, Addis Ababa, Ethiopia, 2017.

[8] World Health Organization, Malaria Report 2010, World Health Organization, Geneva, Switzerland, 2011.

[9] A. J. Rodríguez-Morales, R. A. Barbella, C. Case et al., "Intestinal parasitic infections among pregnant women in Venezuela," Infectious Diseases in Obstetrics and Gynecology, vol. 2006, Article ID 23125, , 2006.

[10] H. Mamo, "Parasitic infections among prison inmates and tobacco farm workers in ShewaRobit, North-Central Ethiopia," PLoS One, vol. 9, no. 6, Article ID e99559, 2014.

[11] O. Hannah, O. A. O. Dada-Adegbola, and O. Catherine, "Asymptomatic malaria and intestinal helminth co-infection among children in a rural community in Southwest Nigeria," Malaria World Journal, vol. 4, pp. 2214-4374, 2013.

[12] A. Degarege, A. Animut, M. Legesse, and B. Erko, "Malaria and helminth co-infections in outpatients of Alaba Kulito Health Center, Southern Ethiopia: a cross sectional study," BMC Research Notes, vol. 3, no. 1, p. 143, 2010.

[13] G. Tadesse, "The prevalence of intestinal helminthic infections and associated risk factors among school children in Babile town, Eastern Ethiopia," Ethiopian Journal of Health Development, vol. 9, no. 2, pp. 140-147, 2005.

[14] M. Legesse and B. Erko, "Prevalence of intestinal parasites among school children in a rural area close to south east of Lake Langano, Ethiopia Ethiopian," Ethiopian Journal of Health Development, vol. 18, no. 2, pp. 116-120, 2004.

[15] B. M. G. Erko, "Human helminthiasis in Wendo Genet, southern Ethiopia with emphasis on geohelminthiasis," Ethiopian Medical Journal, vol. 41, pp. 333-344, 2003.

[16] S. M. Kinung'hi, P. Magnussen, G. M. Kaatano, C. Kishamawe, and B. J. Vennervald, "Malaria and helminth co-infections in school and preschool children: a cross-sectional study in Magu district, north-western Tanzania," PLoS One, vol. 9, no. 1, Article ID e86510, 2014.

[17] C. Njua-Yafi, E. A. Achidi, and J. K. Anchang-Kimbi, "Malaria, helminths, co-infection and anaemia in a cohort of children from Mutengene, south western Cameroon," Malaria Journal, vol. 15, no. 1, p. 69, 2016.

[18] H. K. L. E. Kimbi, S. Wanji, J. V. Mbuh, and J. L. N. Nyanga, "Co-infections of asymptomatic malaria and soil-transmitted helminths in school children in localities with different levels of urbanization in the mount Cameroon region," Journal of Bacteriology \& Parasitology, vol. 3, p. 134, 2012.

[19] G. N. P. Wokem, C. Chukwu, and C. Benjamin Nwachukwu, "Prevalence of intestinal parasites seen in HIV sero-positive subjects in Port Harcourt, Nigeria," Nigerian Journal of Parasitology, vol. 29, no. 2, 2008.

[20] T. Akiyama, T. Pongvongsa, and S. Phrommala, "Asymptomatic malaria, growth status, and anaemia among children in Lao People's Democratic Republic: a cross-sectional study," Malaria Journal, vol. 15, no. 1, p. 499, 2016.

[21] J. L. Abbate, V. O. Ezenwa, J. F. Guégan, M. Choisy, M. Nacher, and B. Roche, "Disentangling complex parasite interactions: protection against cerebral malaria by one helminth species is jeopardized by co-infection with another," PLoS Neglected Tropical Diseases, vol. 12, no. 5, Article ID e0006483, 2018.

[22] A. Teklemariam, M. Alemseged, and S. Adugna, "Malariaintestinal helminthes co-infection among patients in wolkite health center and Attat hospital, gurage zone, southern Ethiopia," Journal of Parasitology and Vector Biology, vol. 10, no. 2, pp. 26-32, 2018.

[23] H. Helmby and Q. Bickle, "Immune modulation by helminth infections," Parasite Immunology, vol. 28, no. 10, pp. 479-481, 2006.

[24] J. D. Turner, H. Faulkner, and J. Kamgno, "Th2 cytokines are associated with reduced worm burdens in a human intestinal helminth infection," The Journal of Infectious Diseases, vol. 188, no. 11, pp. 1768-1775, 2003.

[25] M. Nacher, F. Gay, and D. Chindanond, "Helminth infections are associated with protection from cerebral malaria and increased nitrogen derivatives concentrations in Thailand," The American Journal of Tropical Medicine and Hygiene, vol. 66, no. 3, pp. 304-309, 2002.

[26] WHO, Microscopy for the Detection, identification and Quantification of Malaria Parasites on Stained Thick and Thin Blood Films in Research Settings, WHO, Geneva, Switzerland, 2015.

[27] WHO, Malaria Microscopy Quality Assurance.Manual Version 2, World Health Organization, Geneva, Switzerland, 2016.

[28] F. Odhiambo, A. M. Buff, and C. Moranga, "Factors associated with malaria microscopy diagnostic performance following a pilot quality-assurance programme in health facilities in malaria low-transmission areas of Kenya, 2014," Malaria Journal, vol. 16, no. 1, p. 371, 2017.

[29] B. Ngasala, F. Matata, R. Mwaiswelo, and B. P. Mmbando, "Anemia among schoolchildren with malaria and soiltransmitted helminth coinfections after repeated rounds of mass drug administration in muheza district, Tanzania," The American Journal of Tropical Medicine and Hygiene, vol. 101, no. 5, pp. 1148-1155, 2019.

[30] M. Parker, Z. Han, and E. Abu-Haydar, "An evaluation of hemoglobin measurement tools and their accuracy and reliability when screening for child anemia in Rwanda: a randomized study," PLoS One, vol. 13, no. 1, Article ID e0187663, 2018.

[31] P. Seguin, A. Kleiber, C. Chanavaz, J. Morcet, and Y. Mallédant, "Determination of capillary hemoglobin levels using the HemoCue system in intensive care patients," Journal of Critical Care, vol. 26, no. 4, pp. 423-427, 2011.

[32] WHO, The Global Prevalence of Anaemia in 2011, WHO, Geneva, Switzerland, 2015.

[33] A. Abossie, A. Bekele Kassahun, T. Yohanes, and A. Abera, "Prevalence of asymptomatic Plasmodium falciparium and Plasmodium vivax malaria carriage among school children of malaria endemic areas of Mirab Abaya district, Southern Ethiopia," Journal of Parasitology and Vector Biology, vol. 9, pp. 1-7, 2017.

[34] K. Etienne Angora, D. Vincent, and A. Konaté, "Uncomplicated malaria and intestinal helminth co-infection among Schoolchildren in Abobo District, Abidjan (Côte d' Ivoire)," Journal of Infectious Diseases and Epidemiology, vol. 5, 2018.

[35] M. A. Fikru Gashaw and B. Ero, "Prevalence of intestinal helminthes infection among school children in Maksegnit and Enfranz towns, Northwestern Ethiopia, with emphasis on shistosoma mansoni infection," Parasite and Vectors, vol. 8, 2015. 
[36] S. B. Tsega Teshale and G. Teklay, "Prevalence of intestinal helminthes and associated factors among school children of Medebay Zana Wereda; North Western Tigray, Ethiopia," BMC Researh Notes, vol. 11, p. 444, 2017.

[37] O. Ojurongbe, A. M. Adegbayi, O. S. Bolaji, A. A. Akindele, O. A. Adefioye, and O. A Adeyeba, "Asymptomatic falciparum malaria and intestinal helminths co-infection among school children in Osogbo, Nigeria," Journal of Research in Medical Sciences: The Official Journal of Isfahan University of Medical Sciences, vol. 16, no. 5, pp. 680-686, 2011.

[38] L. S. A. M. Garcia and E. Kokoskin, "Practical guidance for clinical microbiology laboratories: laboratory diagnosis of parasites from the gastrointestinal tract," Clinical Microbiology Reviews, vol. 31, no. 1, Article ID e00025, 2017. 